\title{
ESTIMATIVA DO FLUXO DO CALOR SENSÍVEL UTILIZANDO O ALGORITMO SEBAL E IMAGENS MODIS PARA A REGIÃO NORTE FLUMINENSE, RJ
}

\section{JOSÉ CARLOS MENDONÇA ${ }^{1}$, ELIAS FERNANDES DE SOUSA², ROMÍSIO GERALDO BOUHID ANDRÉ $^{3}$, BERNARDO BARBOSA DA SILVA ${ }^{4}$, NELSON DE JESUS FERREIRA ${ }^{5}$}

${ }^{1}$ Laboratório de Meteorologia, Universidade Estadual Norte Fluminense (LAMET/UENF), Macaé, RJ, Brasil

${ }^{2}$ Laboratório de Engenharia Agrícola (LEAG/UENF), Campos dos Goytacazes, RJ, Brasil

${ }^{3}$ Instituto Nacional de Meteorologia (INMET), Brasília, DF, Brasil

${ }^{4}$ Departamento de Ciências Atmosféricas, Universidade Federal de Campina Grande (DCA/UFCG), Campina Grande, $\mathrm{PB}$, Brasil

${ }^{5}$ Centro de Previsão de Tempo e Estudos Climáticos, Instituto Nacional de Pesquisas Espaciais (CPTEC/INPE), São José dos Campos, SP, Brasil

mendonca@uenf.br, efs@uenf.br, romisio.andre@inmet.gov.br, bernardo@dca.ufcg.edu.br, nelson@cptec.inpe.br

Recebido Agosto de 2010 - Aceito Agosto de 2011

\section{RESUMO}

Neste estudo foram utilizadas imagens do sensor MODIS e o SEBAL na avaliação de duas proposições para a estimação do fluxo de calor sensível (H), baseadas na seleção dos pixels âncoras utilizados na determinação da diferença da temperatura à superfície (dT). Denominou-se H-CLÁSSICO, a proposição que utilizou pixels com temperaturas extremas, e H-PESAGRO, aquela que adotou o pixel frio para a menor temperatura e o pixel quente para o valor de $\mathrm{H}$ obtido como resíduo da equação de Penman-Monteith (FAO56), estimado com dados observados em uma estação agrometeorológica. Os resultados de $\mathrm{H}$ estimados pelas duas proposições foram comparados com valores de $\mathrm{H}$ obtidos pelo Balanço de Energia (Razão de Bowen) sobre uma área cultivada com cana-de-açúcar. Com os resultados obtidos pode-se concluir que a proposição H-PESAGRO necessitou de um menor número de interações para a estabilização dos valores da resistência aerodinâmica $\left(\mathrm{r}_{\mathrm{ah}}\right)$ e que os resultados, estimados com a proposição H-CLÁSSICA, apresentaram valores 58,35\% mais elevados do que os estimados pela H-PESAGRO. Quando comparados com os valores de $\mathrm{H}$ estimados pelo método da razão de Bowen sobre o pixel da cana-de-açúcar, os coeficientes de correlação foram $r=0,54$ e $r=$ 0,71, respectivamente, para as proposições H-CLÁSSICA e H-PESAGRO.

Palavras-chave: sensoriamento remoto, balanço de energia, agrometeorologia, SEBAL

\begin{abstract}
ESTIMATIVE OF SENSIBLE HEAT FLUX (H) IN NORTH FLUMINENSE REGION, RJ, USING MODIS PRODUCTS AND SEBAL ALGORITHM

Images from the MODIS and SEBAL algorithm were used to evaluate two proposals for estimating sensible heat flux $(\mathrm{H})$ based on the selection of anchor pixels used to determine the surface temperature difference (dT). The proposition in which pixels with extreme temperatures were used was called H-CLASSIC. The other one H-PESAGRO adopted for cold pixels the lowest temperature and for the hot pixels the value of $\mathrm{H}$ as a residue of the equation of Penman-Monteith FAO 56, using observed data from agrometeorological station. The results showed that the H-PESAGRO required a smaller number of interactions for the stabilization of the aerodynamic resistance $\left(\mathrm{r}_{\mathrm{ah}}\right)$ values. H- CLASSIC estimated values were $58.35 \%$ higher than the estimated by H-PESAGRO. The estimated $\mathrm{H}$ values using Bowen ratio when compared with the sugar cane pixels presented correlation coefficients of $r$ $=0.54$ and $\mathrm{r}=0.71$ for the Propositions H-CLASSIC and H-PESAGRO, respectively.
\end{abstract}

Keywords: remote sensing, energy balance, agrometeorology, SEBAL 


\section{INTRODUÇÃO}

A radiação solar é a energia utilizada nos processos de aquecimento e resfriamento do ar e do solo, na transferência de vapor d'água da superfície para a atmosfera e no metabolismo das plantas e dos animais. Dada a sua importância, faz-se necessário estudar a partição dos componentes do balanço de energia à superfície e, sobretudo, aqueles relacionados com as perdas de água e o aquecimento da atmosfera (Ferreira, 2006).

Liou (1992) cita que da radiação solar incidente em uma superfície vegetada, parte é refletida de volta para o espaço, outra parte é absorvida pelo dossel e pelo solo, contribuindo para o seu aquecimento, e a terceira parte é transportada para a atmosfera pelos processos de trocas turbulentas que envolvem o dossel e a atmosfera, sendo o saldo de radiação à superfície, o resultado do balanço entre os fluxos radiativos de onda curta e onda longa, ou seja, o balanço entre a radiação global incidente e a radiação global refletida.

O Surface Energy Balance Algorithm for Land (SEBAL) foi desenvolvido e validado por Bastiaanssen (1995) em campanhas experimentais na Espanha e no Egito usando imagens do satélite Landsat 5-TM. Com o SEBAL se obtém o fluxo de calor latente $-\lambda \mathrm{E}\left(\mathrm{W} \mathrm{m}^{-2}\right)$ como resíduo do balanço de energia, o que ocorre após a obtenção do saldo de radiação - Rn $\left(\mathrm{W} \mathrm{m}^{-2}\right)$, fluxo de calor no solo $-\mathrm{G}\left(\mathrm{W} \mathrm{m}^{-2}\right)$ e fluxo de calor sensível - $\mathrm{H}\left(\mathrm{W} \mathrm{m}^{-2}\right)$, nessa ordem. Este algoritmo tem sido aplicado em vários países, e sua ampla utilização se deve ao fato de que o mesmo requer apenas imagens digitais de sensores que possibilitem a determinação do albedo, índices de vegetação e temperatura da superfície, caso dos sensores TM - Landsat 5, MODIS - Terra, AVHRR - NOAA, ASTER - Terra, e poucos dados de superfície. (Allen et al., 2002; Tasumi et al. 2008).

Silva e Bezerra (2006) destacam que H é a parte essencial do SEBAL e é obtido através de processo iterativo, que se inicia considerando a atmosfera em condições de equilíbrio neutro, que serve apenas como valor inicial ao processo iterativo, mas na sequência é identificada a condição de estabilidade, usando como parâmetro a razão z/L, onde L é o comprimento de Molin-
Obukhov. Este parâmetro entra nas equações que permitem as correções dos valores da resistência aerodinâmica ao transporte de calor $\left(\mathrm{r}_{\mathrm{ah}}, \mathrm{s} \mathrm{m}^{-1}\right)$.

Um grande problema relacionado ao emprego do SEBAL, em regiões de clima úmido ou sub-úmido, consiste na identificação do pixel quente, que dificilmente reuniria a condição de estar seco, ou seja, possuir $\lambda E=0$. Neste trabalho é apresentada uma alternativa, denominada de H-PESAGRO, que consiste em considerar como "pixel quente" um local com medições agrometeorológicas de superfície, que possibilitam determinar o valor de $\mathrm{H}$.

Desta forma, este trabalho teve por objetivo, utilizando o algoritmo SEBAL e imagens MODIS, avaliar o desempenho das proposições H-CLÁSSICA e H-PESAGRO para a estimação do fluxo de calor sensível $(\mathrm{H})$ em comparação com $\mathrm{H}$, obtido pelo método do Balanço de Energia (razão de Bowen) sobre uma área cultivada com cana-de-açúcar na região Norte Fluminense, RJ.

\section{MATERIAL E MÉTODOS}

A região Norte Fluminense do estado do Rio de Janeiro possui um clima do tipo tropical úmido (Aw), segundo a classificação de Köppen, com verão chuvoso e inverno seco, sendo a precipitação pluviométrica média anual em torno de 1.023 mm (Gomes, 1999). Na Figura 1 é apresentada a área de estudo.

Foram utilizados os produtos MOD09 e MYD09 (Reflectância de Superfície - GHK e GQK) e MOD11 e MYD11 (Temperatura de Superfície) referentes a 24 cenas sobre o "tile" h14/v11 imageadas pelos sensores MODIS/Terra e MODIS/ Aqua, nos dias de ordem do ano ("Julianos") 218, 227, 230, $241,255,285,320$ e 339 do ano de 2005 e 15, 36, 63, 102, 116, 139, 166, 186, 189, 190, 191, 200, 201, 205, 208 e 221 do ano de 2006. Esses dias foram selecionados por apresentar condições de céu claro.

As imagens foram obtidas gratuitamente junto ao Land Processes Distributed Active Archive Center (LP-DAAC), da National Aeronautics and Space Administration (NASA), no seguinte endereço: http://edcimswww.cr.usgs.gov/pub/

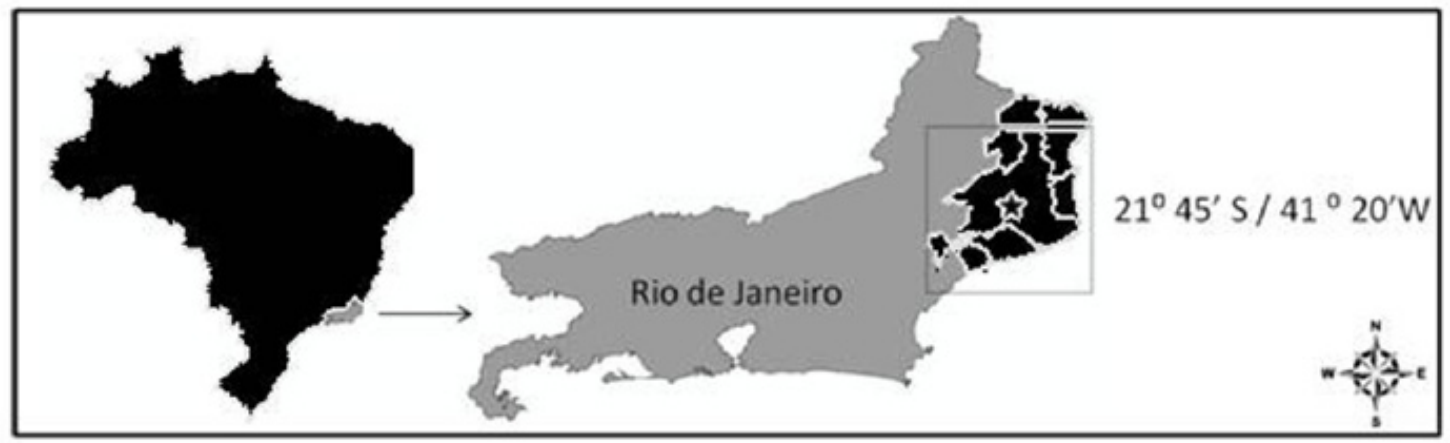

Figura 1 - Localização da área de estudo. 
imswelcome/. As imagens foram tratadas e reordenadas para a resolução espacial de $250 \mathrm{~m}$ utilizando-se a ferramenta MODIS Reprojection Tool (MRT). Para os empilhamentos, composições, recortes e álgebras das imagens utilizou-se o software Erdas Image - Pro, Versão 8.7, sendo os mapas temáticos gerados no aplicativo ArcGis 9.1.

Os dados meteorológicos complementares foram obtidos na Estação Evapotranspirométrica da Universidade Estadual do Norte Fluminense Darcy Ribeiro (UENF), localizada na Estação Experimental de Campos da Empresa de Pesquisa Agropecuária do Estado do Rio de Janeiro, PESAGRO-RIO (21 ${ }^{\circ} 24^{\prime} 48^{\prime \prime}$ S; 41 44' 48" W; h = 14 m). Nela encontra-se uma Estação Meteorológica automática modelo Thies Clima, instalada sobre grama Batatais (Paspalum Notatum) e dotada de anemômetro, barômetro, termohigrômetro, piranômetro e pluviômetro. Todos os sensores são conectados a um coletor de dados modelo DL 15 - V. 2,00 - Thies Clima, registrando valores a cada minuto, sendo armazenado um valor médio a cada 10 minutos. Foram também, obtidos dados em uma Estação Micrometeorológica instalada em uma área ocupada com cana-de-açúcar $\left(21^{\circ} 43^{\prime} 21,8^{\prime \prime} \mathrm{S}\right.$; $41^{\circ} 24^{\prime} 26,1^{\prime \prime} \mathrm{W} ; \mathrm{h}=12 \mathrm{~m}$ ) dotada dos seguintes sensores: 1 saldo radiômetro NR Lite, 2 piranômetros LI 200 X-Li-cor; 2 termohigrômetros HMP45C-L, da Vaissala; 2 anemômetros Met One, da Campbell Scientific e 3 fluxímetros HFP01SC-L, da Hukseflux. Todos os dados desses sensores foram coletados a cada minuto, e extraídos e armazenados os valores médios a cada 15 min num sistema de aquisição de dados CR21X, da Campbell Scientific. As hastes horizontais foram dispostas a $0,50 \mathrm{~m}$ sobre o dossel da cultura ( $1^{\text {a }}$ haste) e a 2,0 m entre a $1^{\text {a }}$ e $2^{\mathrm{a}}$ haste. Esse padrão de coleta de dados sobre o dossel da cana-de-açúcar foi mantido durante todo o ciclo de cultivo, sendo, para tanto, efetuado o reposicionamento do conjunto de hastes sempre que necessário.

No SEBAL, o H foi obtido pela seguinte equação:

$$
\mathrm{H}=\rho \cdot \mathrm{c}_{\mathrm{p}} \cdot \mathrm{dT} / \mathrm{r}_{\mathrm{ah}}
$$

onde $\rho\left(\mathrm{kg} \mathrm{m}^{-3}\right)$ é massa específica do ar, $\mathrm{c}_{\mathrm{p}}\left(1004 \mathrm{~J} \mathrm{~kg}^{-1} \mathrm{~K}^{-1}\right)$ é o calor específico do ar à pressão constante, $\mathrm{dT}$ é a diferença de temperatura $\left(\mathrm{T}_{1}-\mathrm{T}_{2}\right)$ entre as duas alturas $z_{1}$ e $z_{2}$, e $\mathrm{r}_{\mathrm{ah}}$ é a resistência aerodinâmica ao transporte de calor sensível $\left(\mathrm{s} \mathrm{m}^{-1}\right)$.

Inicialmente, $\mathrm{H}$ foi estimado com base na velocidade do vento e na temperatura da superfície, usando-se uma calibração interna da diferença da temperatura entre dois níveis próximos à superfície.

A distribuição espacial da massa específica do ar $(\rho)$, em $\mathrm{kg} \mathrm{m}^{-3}$, visualizada no formato de um mapa temático, foi estimada segundo a metodologia proposta por Allen et al. (1998):

$$
\rho_{a r}=\frac{1000 * P}{1.01 * T s * 287}
$$

onde: $\mathrm{P}$ é a pressão atmosférica do ar, em $\mathrm{kPa}$, dada por:

$$
P=101,3 *\left(\frac{T s-0,0065 z}{T s}\right)^{5,26}
$$

em que: $z$ é a elevação local, em m, e Ts o mapeamento da temperatura da superfície.

A velocidade de fricção $\mathrm{u}_{*}\left(\mathrm{~m} \mathrm{~s}^{-1}\right)$ foi calculada usando-se o perfil logarítmico do vento, considerando-se a condição de neutralidade atmosférica dado por:

$$
u_{*}=\frac{u_{x} k}{\ln \left(\frac{z_{x}}{z_{o m}}\right)}
$$

onde: $\mathrm{u}_{\mathrm{x}}$ é a velocidade do vento $\left(\mathrm{m} \mathrm{s}^{-1}\right)$ na altura $\mathrm{z}_{\mathrm{x}}, \mathrm{k}$ é constante de von Karman = 0,41 (Silva Filho et al., 1992) e $z_{\text {om }}$ é o parâmetro de rugosidade estimado pelo valor da altura média da vegetação existente no entorno da área da estação meteorológica e é calculado pela equação apresentada por Brutsaert (1982):

$$
z_{\text {om }}=0,123 \mathrm{~h}
$$

em que $h(0,30)$ era a altura média da vegetação existente no entorno da estação, em m.

A velocidade do vento medida a uma altura na qual se pode assumir que os efeitos da rugosidade da superfície podem ser desprezados é denominada de blending height, podendo ser obtida pela equação:

$$
u_{100}=u_{*} \frac{\ln \left(\frac{100}{z_{\text {om }}}\right)}{k}
$$

Com a hipótese de que $\mathrm{u}_{100}$ é constante em toda a área, pode-se estimar a velocidade de fricção $u_{*}$ para cada pixel da imagem pela equação:

$$
u_{*}=\frac{u_{100} k}{\ln \left(\frac{100}{z_{o m}}\right)}
$$

onde: $z_{\mathrm{om}}$ foi obtido em função do mapa temático do SAVI (Soil Ajusted Vegetation Index) de acordo com proposta de Bastiaanssen (1995), qual seja:

$$
z_{\text {om }}=\exp (-5,809+5,62 \text { SAVI })
$$

em que: SAVI é o índice de vegetação ajustado para os efeitos do solo.

De posse dos valores de $\mathrm{u}_{*}$ para cada pixel, estimou-se a resistência aerodinâmica $\left(\mathrm{r}_{\mathrm{ah}}\right)\left(\mathrm{em} \mathrm{s} \mathrm{m}^{-1}\right)$ admitindo-se, também, a atmosfera em condições de estabilidade neutra, segundo a equação:

$$
r_{a h}=\frac{\ln \left(\frac{z_{2}}{z_{1}}\right)}{u_{*} k}
$$


onde $r_{a h}$ é a resistência aerodinâmica ao transporte de calor, em s m${ }^{-1}$.

$\mathrm{O}$ cálculo da diferença de temperatura próxima à superfície dT (K) para cada pixel é feito através da relação linear entre dT e a temperatura de cada pixel (Ts):

$$
d T=a+b T s
$$

onde: $a$ e $b$ são coeficientes empíricos, que dependem dos pixels âncoras selecionados (pixels "quente" e "frio").

Para o pixel "frio" assume-se que o fluxo de calor sensível é nulo, ou seja, $\mathrm{H}=0$ e o fluxo do calor latente é obtido por:

$$
\lambda E T_{\text {pixel frio }}=R n-G
$$

Para o pixel "quente" assume-se que o fluxo de calor latente é nulo, ou seja, $\lambda \mathrm{E}=0$, e o fluxo de calor sensível $(\mathrm{H})$ é dado por:

$$
H_{\text {pixel quente }}=R n-G=\frac{\rho c_{p}(a+b T s)}{r_{a h}}
$$

Onde os valores de Ts, Rn, G e $r_{a h}$ são obtidos no pixel quente selecionado.

Com base nos valores de $\mathrm{dT}$ determinados nos pixels quente e frio de cada imagens gera-se o seguinte sistema de equações:

$$
\begin{aligned}
& a+b d T=r_{a h} \frac{(R n-G)}{\rho c p}(\text { pixel quente }) \\
& a+b T s=0(\text { pixel frio })
\end{aligned}
$$

Resolvendo o sistema de Equações 13 e 14, a e b ficam determinados.

Os valores de $\mathrm{H}$ estimados inicialmente pela seleção dos pixels âncoras não representam de forma adequada o fluxo de calor sensível de cada pixel e servem apenas como valores iniciais do processo iterativo, que identifica a condição de estabilidade atmosférica nas iterações subsequentes.

A teoria de Monin-Obukhov descreve um perfil da velocidade vertical, pois considera os efeitos da rugosidade e da estabilidade atmosférica e, portanto, pode ser aplicada para estimar valores corrigidos da $\mathrm{r}_{\mathrm{ah}}$ (Brutsaert, 1982). Utilizou-se, então, essa proposição na identificação da condição de estabilidade atmosférica sendo a estimativa do comprimento de Monin-Obukhov (m) realizada pela seguinte equação:

$$
\mathrm{L}=-\frac{\rho \mathrm{c}_{\mathrm{p}} \mathrm{u}_{*}^{3} \mathrm{~T}_{\mathrm{s}}}{\mathrm{kgH}}
$$

em que todos os termos já foram apresentados, exceto $\mathrm{g}\left(\mathrm{m} \mathrm{s}^{-2}\right)$, que representa o módulo da aceleração da gravidade.

Na Figura 2 é apresentado o fluxograma do processo iterativo para a estabilização de $r_{a h}$ e obtenção do mapa dos valores espacializados do fluxo de calor sensível (H).
$\mathrm{Na}$ estimativa dos valores das correções de estabilidade para o transporte de momentum $\left(\Psi_{\mathrm{m}}\right)$ e do transporte de calor $\left(\Psi_{h}\right)$, as condições atmosféricas (instável, estável e neutra) devem ser consideradas. Foram utilizadas as formulações clássicas de Paulson (1970) e Webb (1970) para ajustar o perfil de vento às condições de estabilidade atmosférica.

Após a estimativa dos valores de $\Psi_{\mathrm{m}}$ e $\Psi_{h}$, pode-se, então, corrigir o valor de $\mathrm{u}_{*}$ para cada pixel da imagem com o uso da seguinte equação:

$$
u_{*}=\frac{k u_{100}}{\ln \left(\frac{100}{z_{\text {om }}}\right)-\psi_{m(100)}}
$$

onde: $\Psi_{\mathrm{m}(100 \mathrm{~m})}$ é o fator de correção para o transporte de momentum obtido em todos os pixels da imagem.

De posse dos valores de $\mathrm{u}_{*}$ corrigidos para cada pixel, obteve-se o mapa espacializado dos valores da resistência aerodinâmica ao transporte de calor sensível corrigido $\left(\mathrm{r}_{\mathrm{ah}}\right)$, sendo:

$$
r_{a h}=\frac{\ln \left(\frac{z_{2}}{z_{1}}\right)-\psi_{h(z 2)}+\psi_{h(z 1)}}{u^{*} k}
$$

onde: $\Psi_{h(z 2)}$ e $\Psi_{h(z 1)}$ são as correções de estabilidade para o transporte de calor a 2,0 m e $0,1 \mathrm{~m}$, respectivamente.

Depois de obtidos os valores desses parâmetros, retorna-se ao cálculo de dT e, na sequência de $\mathrm{H}$, com os novos valores de $r_{a h}$. Esse processo foi repetido diversas vezes até se observar estabilidade nos valores de dT. Adotou-se como critério para conclusão do processo iterativo, o momento em que se observou a repetição dos valores de $r_{a h}$, a partir da segunda casa decimal.

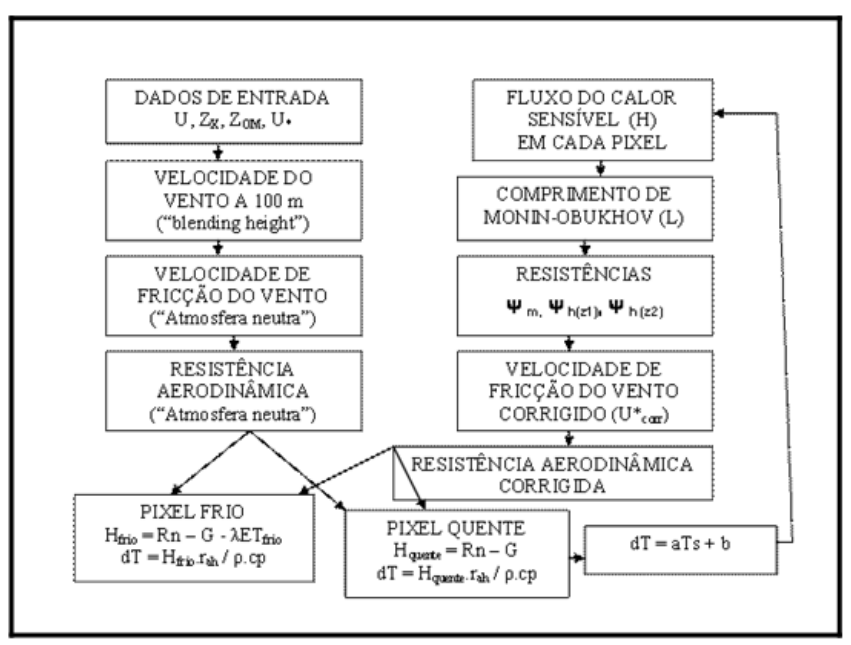

Figura 2 - Fluxograma com as etapas do processo iterativo para estabilização da resistência aerodinâmica, segundo a teoria de MoninObukhov. 
Para condições de baixa velocidade do vento, ou mesmo condições de calmaria, pode ocorrer instabilidade numérica, causada pela limitação dos parâmetros de Monin-Obukov, e assim são gerados erros na estimativa de H. Tasumi (2003) propõe como solução, na ocorrência desses casos, aumentar a velocidade do vento a $100 \mathrm{~m}\left(\mathrm{u}_{100}\right)$ para $4,0 \mathrm{~m} \cdot \mathrm{s}^{-1}$. Nas condições climáticas do Norte Fluminense, RJ, identificou-se essa situação em algumas datas utilizadas neste trabalho, percebendo-se que os baixos valores ocorriam apenas na parte da manhã, englobando o horário de passagem do sensor orbital. Dessa forma, após testar o valor proposto por Tasumi (2003) percebeu-se a permanência do problema. Após diversas avaliações, passou-se, então, a adotar o valor médio diário da velocidade do vento à superfície, acrescido do seu respectivo desvio padrão.

Neste trabalho foram adotadas duas proposições para a determinação dos pixels âncoras, sendo a primeira $\mathrm{H}_{-}$ CLÁSSICO, baseada na seleção de dois pixels com temperaturas extremas. O pixel quente compreendendo uma área de solo exposto ou com pouca vegetação, enquanto o pixel frio foi localizado no interior de um corpo hídrico de grande extensão (Bastiaanssen, 1995).

Baseado na hipótese de que a relação linear entre $T_{\mathrm{s}}$ e dT seria melhor representada a partir da seleção de um pixel quente, onde as componentes do Balanço de Energia à superfície fossem previamente conhecidas, principalmente os valores de $\mathrm{H}$, formulou-se a segunda proposição adotando no critério de seleção do pixel frio a escolha da Lagoa Feia (grande corpo hídrico) e para o pixel quente, adotou-se valores obtidos na estação meteorológica da PESAGRO, onde, através do resíduo da equação de Penman-Monteith (FAO56), foi possível determinar o valor de $\mathrm{H}$, denominado então de H_PESAGRO.

Os valores de $\mathrm{H}$ estimados pelo método da razão de Bowen, com os dados disponíveis e observados numa Estação Micrometeorológica instalada sobre a cultura da cana-de-açúcar, foi pareado e utilizado na comparação com o H estimado pelo SEBAL com as proposições "H-CLASSICA" e "H-PESAGRO".

Para estimativa de $\mathrm{H}$ utilizaram-se, inicialmente, valores de $\mathrm{u}_{2}$ observados na estação meteorológica da PESAGRO. Considerando-se $\mathrm{h}=0,30 \mathrm{~m}$ para a altura da vegetação do entorno da estação meteorológica (grama e culturas anuais), ao se calcular o coeficiente de rugosidade ao transporte de momentum $\left(\mathrm{z}_{\mathrm{om}}\right)$ e, na sequência, determinou-se $\mathrm{u}_{*} \mathrm{e} \mathrm{u}_{100}$.

Na Tabela 1, são apresentados os valores da velocidade do vento observada na estação da PESAGRO, a $2 \mathrm{~m}$ de altura $\left(\mathrm{u}_{2}\right)$, a velocidade de fricção, estimada na estação da PESAGRO $\left(\mathrm{u}_{*}\right)$ e a velocidade do vento a $100 \mathrm{~m}$ de altura, que é considerado constante sobre toda a área de estudo. Todos esses valores são expressos em $\mathrm{m} \mathrm{s}^{-1} \mathrm{e}$ foram utilizados no processamento inicial para gerar as cartas do fluxo de calor sensível $(\mathrm{H})$.

Em que $\mathrm{u}_{2}$ a velocidade do vento a $2 \mathrm{~m}, \mathrm{em} \mathrm{m} \mathrm{s}^{-1} ; \mathrm{u}_{*} \mathrm{a}$ velocidade de fricção, a $2 \mathrm{~m}$, em $\mathrm{m} \mathrm{s}^{-1} ; \mathrm{u}_{100}$ a velocidade do vento a $100 \mathrm{~m}$, também em $\mathrm{m} \mathrm{s}^{-1}$.

Nas Tabelas 2 e 3 são apresentados os valores obtidos no processo interativo para o dia 06/08/2005 (DJ218), na obtenção da estabilidade atmosférica utilizando-se a proposição H-CLÁSSICA e H-PESAGRO.

Na Figura 3 é apresentado o fluxograma elaborado com a ferramenta Model Maker do aplicativo Erdas Image para realização do processamento das imagens e geração dos mapas temáticos utilizados no processo iterativo. O número de iterações e os valores dos coeficientes da regressão linear foram obtidos antecipadamente através de cálculos elaborados numa planilha eletrônica.

Na Tabela 4 são apresentados os valores médios de $\mathrm{H}$, obtidos pela análise estatística dos mapas temáticos, após processo iterativo de estabilização da resistência aerodinâmica ao transporte de calor sensível $\left(\mathrm{r}_{\mathrm{ah}}\right)$, utilizando as duas proposições para seleção dos pixels âncoras.

Tabela 1 - Valores das velocidades do vento observados a $2 \mathrm{~m}$ de altura $\left(\mathrm{U}_{2}\right)$, na estação da Pesagro, da fricção do vento estimada (U*), e a $100 \mathrm{~m}$ de altitude $\left(\mathrm{U}_{100}\right)$, referentes ao instante da passagem do sensor orbital, em $\mathrm{m} \mathrm{s}^{-1}$.

\begin{tabular}{c|cccc}
\hline Data & $\boldsymbol{D J}$ & $\boldsymbol{U}_{\mathbf{2}}$ & $\boldsymbol{U}^{*}$ & $\boldsymbol{U}_{\mathbf{1 0 0}}$ \\
\hline $06 / 08 / 05$ & 218 & 3,40 & 0,35 & 6,73 \\
$15 / 08 / 05$ & 227 & 3,80 & 0,39 & 7,52 \\
$18 / 08 / 05$ & 230 & 4,30 & 0,44 & 8,51 \\
$29 / 08 / 05$ & 241 & 3,70 & 0,38 & 7,33 \\
$12 / 09 / 05$ & 255 & 2,23 & 0,23 & 4,41 \\
$12 / 10 / 05$ & 285 & 3,30 & 0,34 & 6,53 \\
$16 / 11 / 05$ & 320 & 4,90 & 0,50 & 8,85 \\
$05 / 12 / 05$ & 339 & 3,40 & 0,35 & 6,73 \\
$15 / 01 / 06$ & 15 & 2,70 & 0,28 & 5,35 \\
$05 / 02 / 06$ & 36 & 3,60 & 0,37 & 7,13 \\
$04 / 03 / 06$ & 63 & 2,77 & 0,28 & 5,48 \\
$12 / 04 / 06$ & 102 & 2,37 & 0,24 & 4,69 \\
$26 / 04 / 06$ & 116 & 4,22 & 0,43 & 8,35 \\
$19 / 05 / 06$ & 139 & 3,90 & 0,40 & 7,72 \\
$15 / 06 / 06$ & 166 & 2,22 & 0,23 & 4,40 \\
$05 / 07 / 06$ & 186 & 1,89 & 0,19 & 5,86 \\
$08 / 07 / 06$ & 189 & 5,30 & 0,54 & 6,34 \\
$09 / 07 / 06$ & 190 & 4,96 & 0,51 & 9,82 \\
$10 / 07 / 06$ & 191 & 3,26 & 0,34 & 6,45 \\
$19 / 07 / 06$ & 200 & 4,00 & 0,41 & 6,34 \\
$20 / 07 / 06$ & 201 & 2,26 & 0,23 & 4,47 \\
$24 / 07 / 06$ & 205 & 2,89 & 0,30 & 6,53 \\
$27 / 07 / 06$ & 208 & 3,80 & 0,39 & 7,52 \\
$09 / 08 / 06$ & 221 & 4,50 & 0,46 & 8,91 \\
\hline
\end{tabular}




\section{RESULTADOS E DISCUSSÃO}

Observa-se nas Tabelas 2 e 3 que, para o dia 06/08/2005 - DJ218, foram necessárias 10 interações na proposição H-CLÁSSICA e 8 na H-PESAGRO, para que o valor de $r_{a h}$ no pixel quente se repetisse por duas vezes. Comparando-se com os resultados obtidos por Silva et al. (2005), na região de Petrolina, $\mathrm{PE}$, com o sensor TM/Landsat foram necessárias 7 iterações para se obter a estabilidade, enquanto que Tasumi et al. (2003), trabalhando com o mesmo sensor na região oeste dos Estados Unidos, realizaram de 4 a 6 iterações. Neste estudo adotou-se a altura de $200 \mathrm{~m}$ para o cálculo da velocidade do vento livre dos efeitos da rugosidade. Bastiaanssem (1995), em seus trabalhos no norte da África, encontrou entre 3 a 4 iterações necessárias à estabilização dos valores de $\mathrm{r}_{\mathrm{ah}}$.

Neste trabalho, o número de iterações variou entre as proposições, sendo sempre menor em H-PESAGRO. A repetição sucessiva por apenas duas vezes dos valores de $\mathrm{r}_{\mathrm{ah}}$, foi suficiente para aceitar os valores de $\mathrm{H}$ como estáveis, para toda a cena, e que os valores de $\mathrm{L}$ foram inversamente proporcionais aos valores de $\mathrm{dT}$, sendo estes proporcionais à amplitude da temperatura da superfície das cenas.

Para os coeficientes $a$ e $b$, buscou-se trabalhar com número elevado de casas decimais ( 4 para $a$ e 3 para $b$ ), pois os arredondamentos desses coeficientes alteram os valores obtidos no processo iterativo. Quanto aos valores de $\mathrm{u}_{*} \mathrm{e} \mathrm{r}_{\mathrm{ah}}$, nota-se que estes não variaram em ambas as proposições, sendo os valores de $\mathrm{r}_{\mathrm{ah}}$ estimados pelo SEBAL bastante inferiores aos encontrados na bibliografia. Tasumi (2003) relaciona esses baixos valores de $r_{\text {ah }}$ à subestimação que o SEBAL aplica na estimativa do índice de área foliar, enquanto que Bastiaanssen (1995) obteve valores de $\mathrm{r}_{\text {ah }}$ acima de $60 \mathrm{~s} \mathrm{~m}^{-1}$ utilizando o valor de 0,0001 para a altura $\mathrm{z}_{1}$.

Observa-se na Tabela 4, que H-CLASSICA apresentou valores de $\mathrm{H}$ mais elevados que H-PESAGRO. Em valores médios, o H-CLASSICA variou entre 125,4 e 285,7 W.m ${ }^{-2}$, enquanto H-PESAGRO ficou entre 52,2 e 112,4 W.m ${ }^{-2}$. Em valores máximos, a diferença foi ainda maior, pois H-CLASSICA apresentou valores entre 258,6 e 615,4 e H-PESAGRO entre 90,9 e 242,5 $\mathrm{W} \mathrm{m}^{-2}$. Os valores observados na proposição H-CLASSICA estão relacionados à seleção dos pixels âncoras, onde se adotaram os valores extremos de temperatura de superfície. Allen et al. (2002) sugerem que a seleção do pixel quente seja feita em função não só da temperatura da superfície, mas também da relação deste com outros parâmetros, principalmente dos índices de vegetação, tais como o NDVI e o SAVI, e que o pixel frio seja selecionado em uma área bem irrigada, sem déficit hídrico.

Os valores de $\mathrm{H}$ estimados pelo método da razão de Bowen, com os dados disponíveis observados na estação micrometeorológica instalada sobre a cultura da cana-deaçúcar, foram pareados e utilizados na comparação com os de H estimados pelo SEBAL com as proposições "H-CLASSICA" e "H-PESAGRO".

A Figura 4 mostra a correlação entre o $\mathrm{H}$ estimado pelo Balanço de Energia - Razão de Bowen e pelas proposições

Tabela 2 - Valores obtidos no processo iterativo para estabilidade do fluxo de calor sensível (H), no dia 06/08/2005 - DJ218 utilizando a proposição "H CLÁSSICA".

\begin{tabular}{|c|c|c|c|c|c|c|c|c|c|c|c|}
\hline No & $T q(K)$ & $T f(K)$ & $r_{a h \_I N I}$ & $R N(P q)$ & $G(P q)$ & $R N-G(P q)$ & $d T$ & $\overline{b \_}$ & $\bar{a}$ & $\bar{b}$ & H_q \\
\hline 1 & 304,32 & 295,06 & 20,35 & 410,73 & 57,66 & 353,07 & 6,27 & $-295,06$ & 0,6737 & $-198,796$ & 353,07 \\
\hline 2 & 304,32 & 295,06 & 10,56 & 410,73 & 57,66 & 353,07 & 3,24 & $-295,06$ & 0,3498 & $-103,199$ & 353,07 \\
\hline 3 & 304,32 & 295,06 & 14,19 & 410,73 & 57,66 & 353,07 & 4,35 & $-295,06$ & 0,4697 & $-138,593$ & 353,07 \\
\hline 4 & 304,32 & 295,06 & 12,98 & 410,73 & 57,66 & 353,07 & 3,98 & $-295,06$ & 0,4299 & $-126,860$ & 353,07 \\
\hline 5 & 304,32 & 295,06 & 13,39 & 410,73 & 57,66 & 353,07 & 4,10 & $-295,06$ & 0,4432 & $-130,783$ & 353,07 \\
\hline 6 & 304,32 & 295,06 & 13,25 & 410,73 & 57,66 & 353,07 & 4,06 & $-295,06$ & 0,4388 & $-129,481$ & 353,07 \\
\hline 7 & 304,32 & 295,06 & 13,30 & 410,73 & 57,66 & 353,07 & 4,08 & $-295,06$ & 0,4403 & $-129,914$ & 353,07 \\
\hline 8 & 304,32 & 295,06 & 13,28 & 410,73 & 57,66 & 353,07 & 4,07 & $-295,06$ & 0,4398 & $-129,770$ & 353,07 \\
\hline 9 & 304,32 & 295,06 & 13,29 & 410,73 & 57,66 & 353,07 & 4,07 & $-295,06$ & 0,4400 & $-129,818$ & 353,07 \\
\hline 10 & 304,32 & 295,06 & 13,29 & 410,73 & 57,66 & 353,07 & 4,07 & $-295,06$ & 0,4399 & $-129,802$ & 353,07 \\
\hline No & U* ${ }_{\text {INI }}$ & $\mathbf{L}$ & $\mathbf{x} \_100$ & $\mathbf{x} \_2$ & x_0,1 & FI_100 & FI_2 & FI_0,1 & Zom & $\mathrm{U}^{*} \operatorname{corr}$ & $\mathbf{r}_{\text {ah__ }}$ corr \\
\hline 1 & 0,36 & $-11,418$ & 3,447 & 1,396 & 1,033 & 2,455 & 0,777 & 0,067 & 0,046 & 0,528 & 10,56 \\
\hline 2 & 0,53 & $-36,245$ & 2,592 & 1,171 & 1,011 & 1,687 & 0,341 & 0,022 & 0,046 & 0,460 & 14,19 \\
\hline 3 & 0,46 & $-24,038$ & 2,867 & 1,236 & 1,016 & 1,947 & 0,468 & 0,032 & 0,046 & 0,481 & 12,98 \\
\hline 4 & 0,48 & $-27,455$ & 2,775 & 1,213 & 1,014 & 1,861 & 0,423 & 0,029 & 0,046 & 0,474 & 13,39 \\
\hline 5 & 0,47 & $-26,259$ & 2,805 & 1,220 & 1,015 & 1,890 & 0,438 & 0,030 & 0,046 & 0,476 & 13,25 \\
\hline 6 & 0,48 & $-26,650$ & 2,795 & 1,218 & 1,015 & 1,881 & 0,433 & 0,029 & 0,046 & 0,475 & 13,30 \\
\hline 7 & 0,48 & $-26,519$ & 2,798 & 1,219 & 1,015 & 1,884 & 0,435 & 0,030 & 0,046 & 0,476 & 13,28 \\
\hline 8 & 0,48 & $-26,563$ & 2,797 & 1,219 & 1,015 & 1,883 & 0,434 & 0,029 & 0,046 & 0,476 & 13,29 \\
\hline 9 & 0,48 & $-26,548$ & 2,798 & 1,219 & 1,015 & 1,883 & 0,434 & 0,029 & 0,046 & 0,476 & 13,29 \\
\hline 10 & 0,48 & $-26,553$ & 2,798 & 1,219 & 1,015 & 1,883 & 0,434 & 0,029 & 0,046 & 0,476 & 13,29 \\
\hline
\end{tabular}


Tabela 3 - Valores obtidos no processo iterativo para estabilidade do fluxo de calor sensível (H), no dia 06/08/2005 - DJ218 utilizando a proposição H - PESAGRO".

\begin{tabular}{|c|c|c|c|c|c|c|c|c|c|c|c|}
\hline No & $T q(K)$ & $T f(K)$ & $r_{a h I N I}$ & $R N(P q)$ & $G(P q)$ & $R N-G(P q)$ & $d T$ & b_o & $\bar{a}$ & $b$ & $H_{-} q$ \\
\hline 1 & 300,68 & 295,06 & 19,00 & 439,02 & 49,44 & 389,58 & 1,86 & $-295,06$ & 0,3310 & $-97,679$ & 114,14 \\
\hline 2 & 300,68 & 295,06 & 13,45 & 439,02 & 49,44 & 389,58 & 1,32 & $-295,06$ & 0,2343 & $-69,136$ & 114,14 \\
\hline 3 & 300,68 & 295,06 & 15,13 & 439,02 & 49,44 & 389,58 & 1,48 & $-295,06$ & 0,2637 & $-77,804$ & 114,14 \\
\hline 4 & 300,68 & 295,06 & 14,69 & 439,02 & 49,44 & 389,58 & 1,44 & $-295,06$ & 0,2559 & $-75,514$ & 114,14 \\
\hline 5 & 300,68 & 295,06 & 14,81 & 439,02 & 49,44 & 389,58 & 1,45 & $-295,06$ & 0,2581 & $-76,142$ & 114,14 \\
\hline 6 & 300,68 & 295,06 & 14,77 & 439,02 & 49,44 & 389,58 & 1,45 & $-295,06$ & 0,2575 & $-75,972$ & 114,14 \\
\hline 7 & 300,68 & 295,06 & 14,78 & 439,02 & 49,44 & 389,58 & 1,45 & $-295,06$ & 0,2576 & $-76,018$ & 114,14 \\
\hline 8 & 300,68 & 295,06 & 14,78 & 439,02 & 49,44 & 389,58 & 1,45 & $-295,06$ & 0,2576 & $-76,005$ & 114,14 \\
\hline No & $\mathbf{U}^{*}{ }_{\text {INI }}$ & $\mathbf{L}$ & $\mathbf{x} \_100$ & $\mathbf{x} \_2$ & $\mathbf{x} \_\mathbf{0 , 1}$ & FI_100 & FI_2 & FI_0,1 & Zom & $\mathbf{U}^{*}$ corr & $\mathbf{r}_{\mathrm{ah}} \operatorname{corr}$ \\
\hline 1 & 0,39 & $-43,56$ & 2,48 & 1,15 & 1,01 & 1,58 & 0,29 & 0,02 & 0,077 & 0,49 & 13,45 \\
\hline 2 & 0,49 & $-91,65$ & 2,07 & 1,08 & 1,00 & 1,16 & 0,16 & 0,01 & 0,077 & 0,46 & 5,13 \\
\hline 3 & 0,46 & $-73,94$ & 2,18 & 1,09 & 1,01 & 1,27 & 0,19 & 0,01 & 0,077 & 0,47 & 14,69 \\
\hline 4 & 0,47 & $-78,30$ & 2,15 & 1,09 & 1,01 & 1,24 & 0,18 & 0,01 & 0,077 & 0,47 & 14,81 \\
\hline 5 & 0,47 & $-77,08$ & 2,16 & 1,09 & 1,01 & 1,25 & 0,18 & 0,01 & 0,077 & 0,47 & 14,77 \\
\hline 6 & 0,47 & $-77,41$ & 2,16 & 1,09 & 1,01 & 1,25 & 0,18 & 0,01 & 0,077 & 0,47 & 14,78 \\
\hline 7 & 0,47 & $-77,32$ & 2,16 & 1,09 & 1,01 & 1,25 & 0,18 & 0,01 & 0,077 & 0,47 & 14,78 \\
\hline 8 & 0,47 & $-77,35$ & 2,16 & 1,09 & 1,01 & 1,25 & 0,18 & 0,01 & 0,077 & 0,47 & 14,78 \\
\hline
\end{tabular}

*Em que, para ambas Tabelas 2 e 3: Tq $(\mathrm{K})$ - temperatura do pixel quente, em Kelvin; Tf(K) - temperatura do pixel frio, em Kelvin; rahini - resistência aerodinâmica, em s $\mathrm{m}^{-1} ; \mathrm{RN}$ Pq - saldo de radiação instantâneo do pixel quente, em W m${ }^{-2} ; \mathrm{G} \mathrm{Pq}$ - fluxo de calor no solo no pixel quente, em W $\mathrm{m}^{-2}$; dT - diferença de temperatura, em Kelvin; $\mathrm{b} \_0$ - valor do coeficiente da regressão linear do pixel frio; a e b são - valores dos coeficientes da regressão linear entre dT e a temperatura da superfície (Ts); H_q - valor do fluxo de calor sensível no pixel quente, em W m ${ }^{-2}$; $\mathrm{U}^{*}$ ini - velocidade de fricção do vento no início do processo iterativo, em $\mathrm{m} \mathrm{s}^{-1}$; $\mathrm{L}$ - comprimento de Monin-Obukhov, em m; x_100 m, x_2 m ex_0,1m - valores dos componentes de ajustamentos para 100, 2 e 0,1 m das correções de estabilidade para o momento (fi_100) e para o transporte de calor (Fi_2 e Fi_0,1); Zom - coeficiente de rugosidade do vento; $\mathrm{U}^{*}$ corr - velocidade de fricção do vento corrigida, $\mathrm{em} \mathrm{m} \mathrm{s}^{-1}$; rahcorr - resistência aerodinâmica corrigida, em s.m ${ }^{-1}$.

Tabela 4 - Correlação dos valores do fluxo de calor sensível (H) estimados pela razão de Bowen e pelas proposições "H-CLASSICA" (A) e "H-PESAGRO" (B) sobre o pixel da cana-de-açúcar, em $\mathrm{MJ} \mathrm{m}^{-2} \mathrm{dia}^{-1}$.

\begin{tabular}{l|cccccccc}
\hline DJ & \multicolumn{2}{c}{ Média } & \multicolumn{2}{c}{ Máxima } & \multicolumn{2}{c}{ Mínima } & \multicolumn{2}{c}{ Mediana } \\
& H_Clássica & H_Pesagro & H_Clássica & H_Pesagro & H_Clássica & H_Pesagro & H_Clássica & H_Pesagro \\
\hline 218 & 165,23 & 89,38 & 357,29 & 193,21 & 0,0 & 0,0 & 173,06 & 94,34 \\
227 & 210,76 & 89,26 & 466,80 & 193,99 & 0,0 & 0,0 & 220,64 & 63,96 \\
230 & 236,99 & 106,85 & 505,45 & 224,85 & 0,0 & 0,0 & 252,72 & 115,06 \\
241 & 228,25 & 52,91 & 480,64 & 107,46 & 0,0 & 0,0 & 249,71 & 57,93 \\
255 & 223,59 & 92,32 & 435,44 & 178,32 & 0,0 & 0,0 & 246,63 & 102,40 \\
285 & 258,52 & 91,93 & 555,78 & 193,50 & 0,0 & 0,0 & 293,09 & 104,31 \\
320 & 285,67 & 112,45 & 611,35 & 242,52 & 0,0 & 0,0 & 317,61 & 125,05 \\
339 & 205,07 & 98,85 & 486,21 & 232,50 & 0,0 & 0,0 & 235,51 & 113,53 \\
15 & 229,47 & 55,68 & 556,92 & 130,94 & 0,0 & 0,0 & 261,05 & 63,93 \\
36 & 266,78 & 55,85 & 615,40 & 127,40 & 0,0 & 0,0 & 290,87 & 61,71 \\
63 & 179,57 & 81,97 & 450,02 & 200,59 & 0,0 & 0,0 & 154,70 & 70,52 \\
102 & 174,42 & 85,10 & 446,27 & 216,11 & 0,0 & 0,0 & 174,32 & 86,95 \\
116 & 200,44 & 102,99 & 496,48 & 257,37 & 0,0 & 0,0 & 203,64 & 103,55 \\
139 & 183,23 & 74,29 & 393,67 & 158,78 & 0,0 & 0,0 & 192,22 & 78,77 \\
166 & 125,42 & 74,29 & 291,74 & 158,78 & 0,0 & 0,0 & 128,78 & 78,77 \\
186 & 151,84 & 79,34 & 310,21 & 165,57 & 0,0 & 0,0 & 159,56 & 82,79 \\
189 & 154,23 & 75,15 & 332,84 & 166,23 & 0,0 & 0,0 & 162,52 & 75,32 \\
190 & 176,93 & 52,25 & 310,15 & 90,95 & 0,0 & 0,42 & 191,42 & 56,65 \\
191 & 174,20 & 78,04 & 354,62 & 161,07 & 0,0 & 0,0 & 180,08 & 81,79 \\
200 & 194,15 & 111,19 & 397,15 & 232,19 & 0,56 & 0,47 & 197,31 & 114,52 \\
201 & 136,87 & 108,3 & 258,63 & 203,69 & 0,0 & 0,0 & 146,49 & 116,96 \\
205 & 175,64 & 52,51 & 343,57 & 103,01 & 0,28 & 0,0 & 189,36 & 56,73 \\
208 & 212,84 & 80,84 & 418,43 & 155,48 & 0,0 & 0,0 & 240,27 & 91,10 \\
221 & 196,93 & 75,51 & 430,58 & 163,48 & 0,0 & 0,0 & 211,92 & 81,74 \\
\hline
\end{tabular}




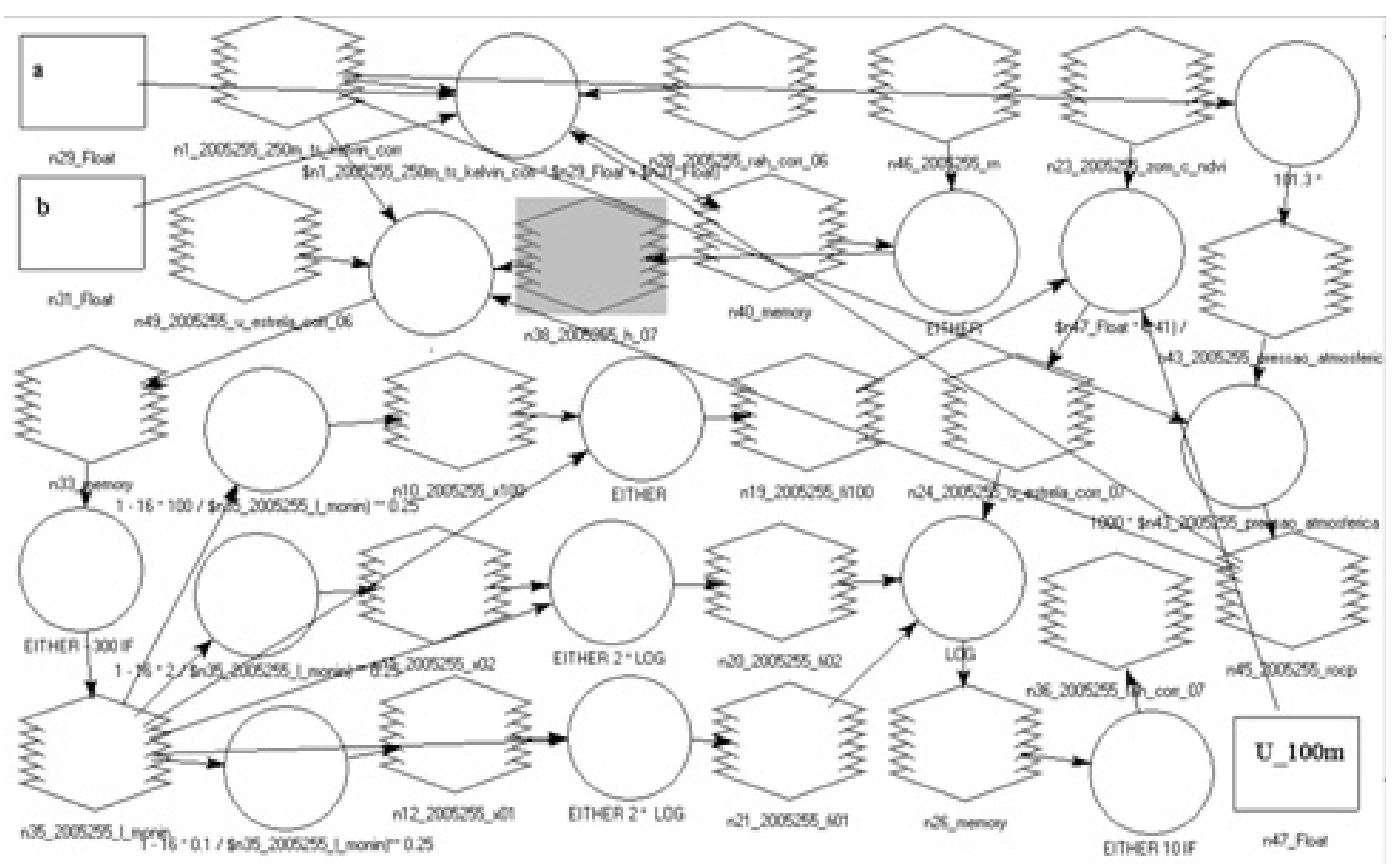

Figura 3 - Fluxograma elaborado pela ferramenta Model Maker do software, Erdas Image ver. 8,6 - Pro, para o processamento iterativo das imagens e geração das cartas do fluxo de calor sensível $(\mathrm{H})$.

"H-CLÁSSICA" (A) e "H-PESAGRO" (B) para o momento da passagem do sensor orbital.

Observa-se na Figura 4 que a proposição H-PESAGRO apresentou um coeficiente de correlação $(r=0,71)$ maior do que a proposição "H-CLASSICA" $(\mathrm{r}=0,54)$, indicando que H-PESAGRO se correlaciona de forma mais linear com a razão de Bowen. Silva et al. (2005) aplicaram o SEBAL em imagens TM/Landsat 5 dos dias 04/12/2000 e 04/10/2000, no perímetro irrigado Nilo Coelho, na região do Sub-Médio São Francisco e observaram valores médios de $\mathrm{H}$ de 106,1, 189,0, 188 e $-18,1 \mathrm{~W} \mathrm{~m}^{-2}$ na imagem de 2000 e $152,2,214,6,200,3$ e $-22,4 \mathrm{~W} \mathrm{~m}^{-2}$, na imagem de 2001, respectivamente para áreas sob pivô central, solo exposto, Caatinga e lago de Sobradinho. Os valores máximos observados pelos mesmos autores foram de $150,5,245,3,260,0$ e $-15,7 \mathrm{~W} \mathrm{~m}^{-2}$ na imagem de $2000 \mathrm{e}$ $231,6,228,0,211,4 \mathrm{e}-20,1 \mathrm{~W} \mathrm{~m}^{-2}$ na imagem de 2001. Paiva

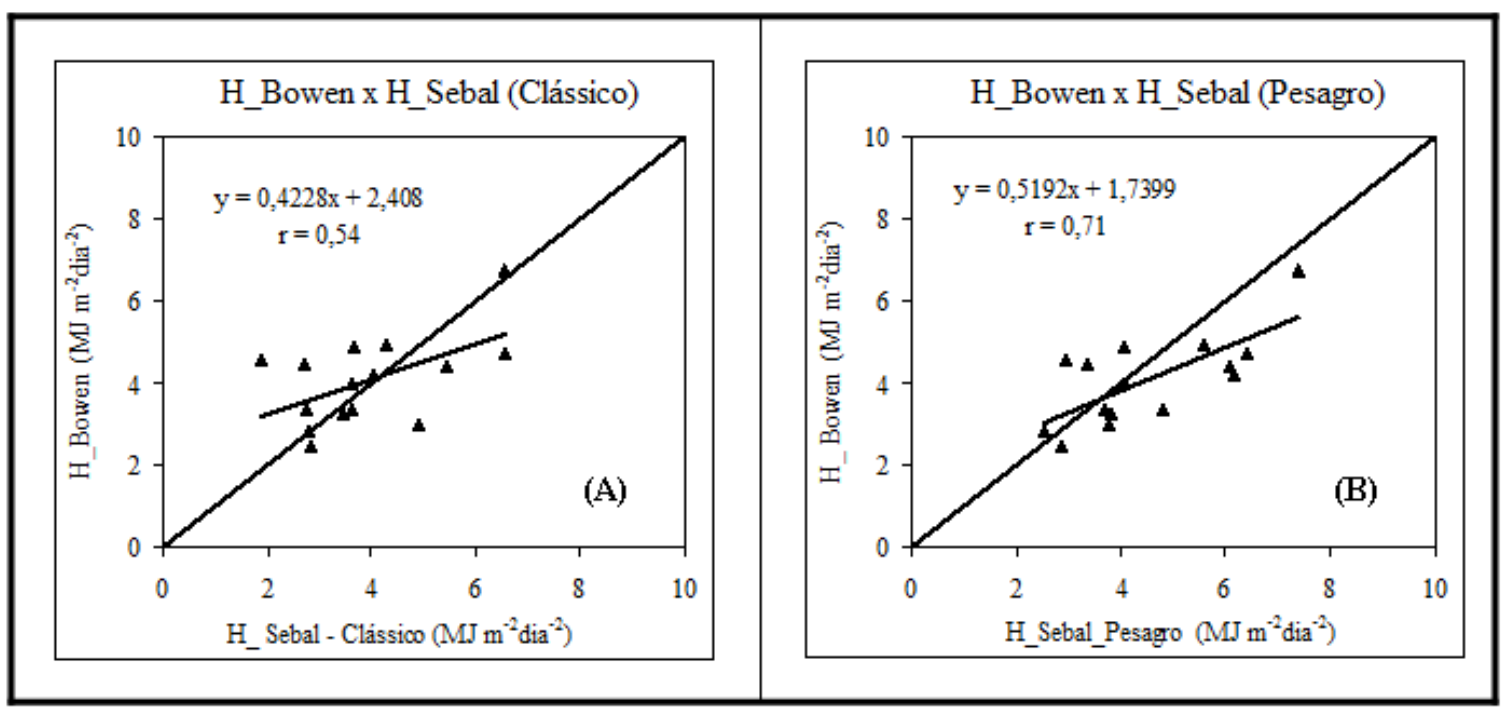

Figura 4 - Correlação dos valores do fluxo de calor sensível (H) estimados pela razão de Bowen e pelas proposições "H-CLASSICA" (A) e "H-PESAGRO" (B) sobre o pixel da cana-de-açúcar, em $\mathrm{MJ} \mathrm{m}^{-2} \mathrm{dia}^{-1}$. 


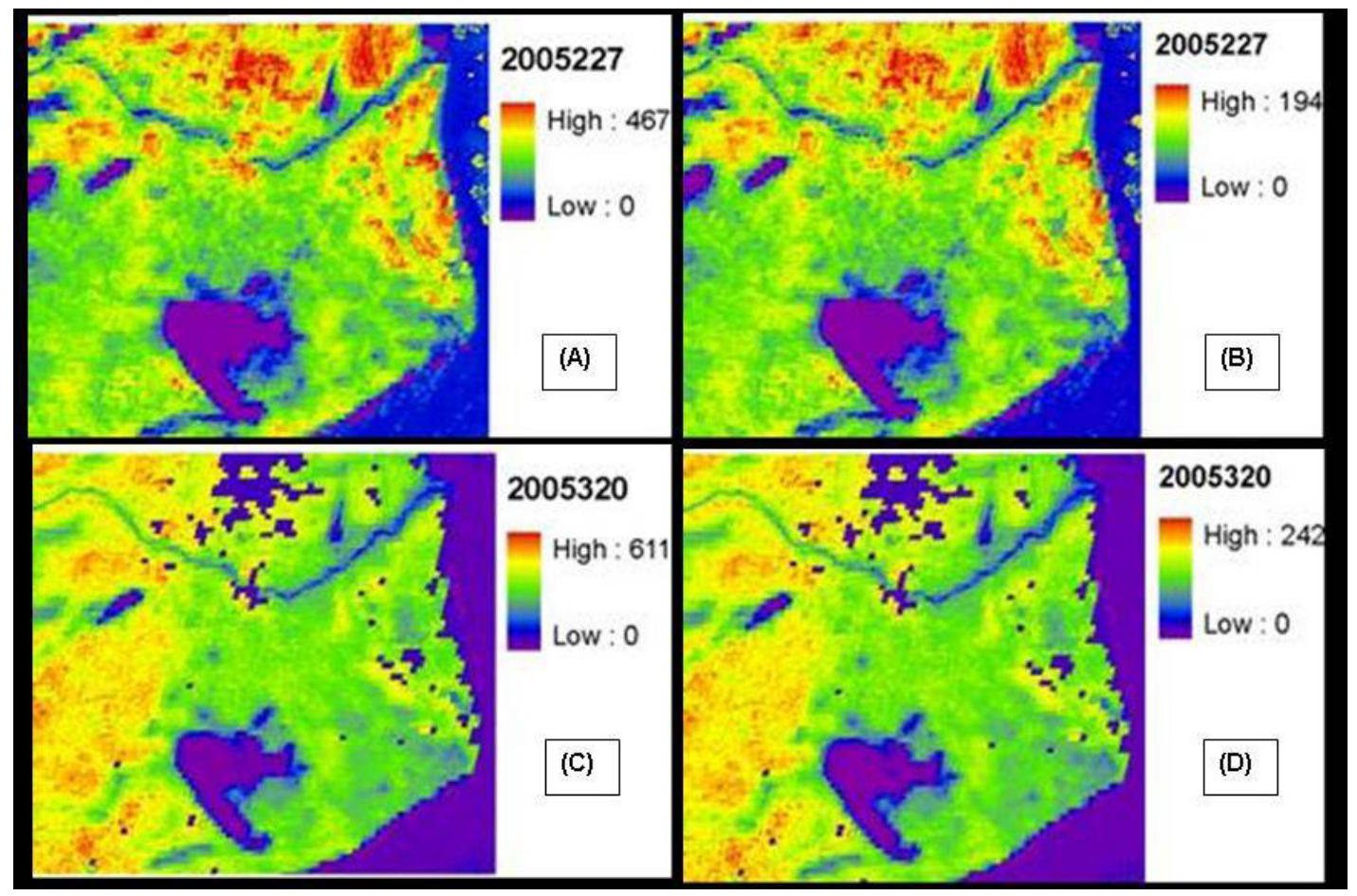

Figura 5 - Variação espacial do fluxo de calor sensível (H) estimados para os dias 15/08/2005 e 16/11/2005, utilizando-se as proposições "H-CLÁSSICA" (A e C) e "H-PESAGRO" (B e D), em W m².

(2005), utilizando imagens do sensor AVHRR/NOOA do dia 16/12/1999, estimou pelo SEBAL, valores médios instantâneos entre 150,78 e 244,54 W m$~^{-2}$, sobre a cultura da soja, na região de Dourados, MT. Ressalta-se que, para os valores obtidos, Silva et al (2005) e Paiva (2005) utilizaram metodologias semelhantes à proposição H-CLÁSSICA.

Na Figura 5 é apresentada a variação espacial do $\mathrm{H}$ estimado pelo SEBAL através das proposições "CLÁSSICA" (A e B) e "H-PESAGRO" (C e D) para os dias 15/08/05 (DJ227) e 16/11/05 (DJ320), em W m ${ }^{-2}$.

\section{CONCLUSÕES}

Em termos médios para todo o período avaliado, os valores de $\mathrm{H}$ utilizando a proposição H-CLÁSSICA apresentaram valores mais elevados que H-PESAGRO (média de 58,35\%). Quando comparados com os valores de $\mathrm{H}$ obtidos pelo método da razão de Bowen, os valores de $\mathrm{H}$ obtidos pela proposição H-PESAGRO apresentaram um coeficiente de correlação mais elevado do que a proposição H-CLASSICA $(\mathrm{r}=0,71$ e $\mathrm{r}=0,54$, respectivamente). Foi necessário um menor número de interações para a estabilização dos valores da resistência aerodinâmica, utilizando-se a proposição H-PESAGRO. Para regiões de climas sub-úmido ou úmido, a proposição H_PESAGRO representa uma alternativa consistente em substituição ao pixel quente e seco exigido pelo SEBAL.

\section{REFERÊNCIAS BIBLIOGRÁFICAS}

Allen, R. G., PEREIRA, L. S., SMIth, M. Crop Evapotranspiration - Guidelines for Computing Crop Water Requirements. FAO Irrigation and Drainage Paper, No. 56, FAO. Rome, Italy, 318 p. 1998.

ALLEN, R.G., TASUMI, M., TREZZA, R., BASTIAANSSEN, W.G.M. SEBAL - Surface Energy Balance Algorithms for Land. Advanced Training and Users Manual, Version 1.0. University of Idaho, EUA. 97 p. 2002.

BASTIAANSSEN, W. G. M. Regionalization of surface flux densities and moisture indicators in composite terrain. Wageningen, The Netherlands: WAU, 1995. 273p. Ph,D Thesis, Wageningen Agricultural University, Wageningen, 1995.

BRUTSAERT, W. Evaporation Into the Atmosphere: Theory, History and Applications. D. Reidel Pub, Co, 299 p. 1982. FERREIRA, A. G. M. Meteorologia Prática. $1^{\mathrm{a}}$ ed. São Paulo: Oficina de Textos, 188 p. 2006.

GOMES, M. C. R. Efeito da irrigação suplementar na produtividade da cana-de-açúcar em Campos dos Goytacazes, RJ. Campos dos Goytacazes, RJ: UENF, 1999. 51p. Dissertação (Mestrado em Produção Vegetal) Universidade Estadual do Norte Fluminense - UENF, 1999. LIOU, K. N. Radiation and Cloud Processes in the Atmosphere: Theory, Observation and Modeling. London: Oxford University Press. 487 p. 1992. 
PAIVA C. M. Estimativa do balanço de energia e temperatura de superfície via satélite NOOA/AVHRR. Rio de Janeiro, RJ: UFRJ. Tese (Doutorado em Engenharia Civil) Universidade Federal do Rido de Janeiro - UFRJ, 218 p 2005.

PAULSON, C.A. The Mathematical Representation of Wind Speed and Temperature Profiles in the Unstable Atmospheric Surface Layer. Journal of Applied Meteorology, v.9, p.856-861, 1970.

SILVA FILHO, V.P., VISWANADHAM, Y., SANTOS, J.M. Determinação da constante de von-Karman a partir de dados coletados na Amazônia. Revista Brasileira de Meteorologia, v.7, n.1, p.535-541, 1992.

SILVA, B.B. da, LOPES, G.M., AZEVEDO, P.V. Balanço de Radiação em Áreas Irrigadas Utilizando Imagens Landsat 5 -TM. Revista Brasileira de Meteorologia, v.20, n.2, p.243-252, 2005.
SILVA, B. B. da, BEZERRA, M. V. C. Determinação dos Fluxos de Calor Sensível e Latente na Superfície Utilizando Imagens TM - Landsat 5. Revista Brasileira de Agrometeorologia, v.13, n.2, p.174-186, 2006.

TASUMI, M. Progress in Operational Estimation of Regional Evapotranspiration Using Satellite Imagery. PhD Dissertation. Idaho State University. Idaho. USA. 379f. 2003.

TASUMI, M., ALLEN, R.G., TREZZA, R. At-Surface Reflectance and Albedo from Satellite for Operational Calculation of Land Surface Energy Balance. Journal Hydrologic Engineering v.13, n.2, p.51-63, 2008.

WEBB, E.K. Profile Relationships: the Log-linear Range and Extension to Strong Stability. Quarterly Journal of the Royal Meteorological Society, v.96, p.67-90, 1970. 